\title{
ON METAL-DIELECTRIC TRANSITION IN LASER ABLATION MODELING
}

\author{
A.A. SAMOKHIN ${ }^{*}$, A.E. ZUBKO ${ }^{1}$ \\ ${ }^{1}$ Prokhorov General Physics Institute of the Russian Academy of Sciences (GPI RAS) \\ Moscow, Russia \\ *Corresponding author. E-mail: asam40@ mail.ru
}

DOI: $10.20948 /$ mathmontis-2019-46-10

Summary. Laser ablation modeling taking into account possibility of metal-dielectric transition in the irradiated metal targets is considered. In the framework of 1-D heat conduction approach it is shown that the steady-state vaporization regime with metaldielectric transition in mercury can be observed only in very narrow laser intensity interval. From analysis of recent experimental results on mercury ablation with nanosecond laser pulses it follows that the results can not be described in the framework of 1-D approach.

\section{INTRODUCTION}

Laser-matter interaction gives possibility to obtain rather high temperature and pressure values which include values of liquid-vapor critical parameters for any element. However critical parameters of most metals remain badly known and this fact is mentioned in many papers [1-4]. From [1] it follows that the difference between critical pressure values for $\mathrm{Al}$ in scientific literature is about an order of magnitude. In ref. [4] one can read:

"Very old and not resolved yet problem of extreme uncertainty in our knowledge in location and critical point parameters for uranium and its compounds $\left(\mathrm{UO}_{2}, \mathrm{UN}, \mathrm{UC} \ldots\right)$ is under discussion. The expected critical region for uranium is not presently achievable experimentally. The same is true for powerful, but laborious contemporary socalled firstprinciple approaches (QMD, QMC etc). At the same time traditional way of theoretical estimations for critical parameters on the base of far extrapolation of well-known lowtemperature thermal and caloric properties for condense phase, lead to extreme dispersion in predicted values for uranium critical temperature and pressure. We discuss possible physical reasons, which could explain and justify mentioned above critical parameters discrepancy for uranium. In particular we discuss possible anomalies in falling of effective "ionization degree" on the expansion way from triple to critical point, as well as possibility for existing of hypothetical additional "entropic" phase transition, similar to that predicted by Landau and Zeldovich long ago."

For this reason, further theoretical and experimental investigations of intense laser-metal interaction are necessary including the possibility of metal-dielectric transition which is also discussed in many papers (see e.g. [4-11] and references therein).

In the present paper possible manifestations of this transition for mercury are analyzed in the framework of simplified one-dimensional approach using the previous results $[7,8]$ and recent experimental investigation [11]. 


\section{1-D HEAT CONDUCTION APPROACH}

\subsection{Steady-state regime of laser ablation}

One dimensional temperature distribution in laser irradiated condensed matter located at $z$ $\geq z_{0} \geq 0$ is described with the heat conduction equation

$$
\frac{\partial T}{\partial t}=\frac{\partial}{\partial z}\left(\chi \frac{\partial T}{\partial z}\right)+\frac{\alpha I}{C} e^{-\alpha\left(z-z_{0}\right)}
$$

where $T, \chi, C, \alpha, I, z_{0}$ are temperature, thermal diffusivity, heat capacity, absorption coefficient, absorbed intensity and irradiated surface position respectively. In (1) hydrodynamic movement is not taken into account, the approximation being applicable to some extent for sufficiently short laser pulses and for the cases when heat expansion can be neglected.

At irradiated surface $z_{0}$ in vacuum vaporization boundary condition is formulated as

$$
\begin{gathered}
\chi \frac{\partial T}{\partial z}=\frac{L V}{C}, \quad T\left(z_{0}\right)=T_{s} \quad \text { at } z=z_{0} \\
V=0.82 \frac{P_{b} \exp \left(A\left(1-T_{b} / T_{s}\right)\right)}{\sqrt{2 \pi m k T_{s}}}
\end{gathered}
$$

where $L, V, T_{s}, T_{b}, P_{b}, k$ are heat of vaporization, vaporization velocity, surface temperature, boiling temperature, boiling pressure and Boltzmann constant respectively; constant $A=11.4$. Boundary condition (2) and vaporization velocity (3) does not change in the moving reference frame for the vacuum vaporization case.

In the reference frame moving together with evaporating surface (the change of reference system is $\left.z=z^{\prime}-\int V d t\right)$ the irradiated surface position is fixed $\left(z_{0}=0\right.$, used below everywhere) and instead of (1) one has

$$
\frac{\partial T}{\partial t}-V \frac{\partial T}{\partial z}=\frac{\partial}{\partial z}\left(\chi \frac{\partial T}{\partial z}\right)+\frac{\alpha I}{C} e^{-\alpha z}
$$

Under conditions $\partial T / \partial t=0$ and $I=$ const this equation yields the steady-state form of the heat conduction equation

$$
\frac{\partial}{\partial z}\left(\chi \frac{\partial T}{\partial z}\right)+V \frac{\partial T}{\partial z}+\frac{\alpha I}{C} e^{-\alpha z}=0
$$

After space integration in (4) from $z_{0}=0$ to $z \rightarrow \infty$ it follows (see [12] and references therein)

$$
I=V\left[L+C\left(T_{s}-T_{\infty}\right)\right]
$$

where $T_{\infty}$ is temperature of unperturbed medium at $z>\alpha^{-1}, z>\chi / V$. Relation (5) is an energy balance equation, and in conjunction with (3) it allows to find $T_{s}$ and $V$ at given $I$. Note that (5) remains valid also for the case of variables $\alpha$ and $\chi$.

The relation (5) does not depend on $\alpha$ and $\chi$, while it is not so for temperature distribution $T(z)$ which at constant $\alpha$ and $\chi$ values is given by the well-known expression (see e.g. [12] and references therein) 


$$
T(z)=T_{\infty}-\left(\frac{L}{C}+\frac{y}{1-y} \frac{I}{C V}\right) e^{-\frac{\alpha z}{y}}+\frac{1}{1-y} \frac{I}{C V} e^{-\alpha z}
$$

The curve $T(z)$ depends significantly on the dimensionless parameter $y=\alpha \chi / V$ as it is seen from Fig. 1 which describes two curves $T(z)$ at different $y$ values $(y=460$ for mercury and $y=$ 1.1 for water) and constant $T_{s} / T_{c} \approx 0.6$ where $T_{c}$ is a critical temperature of liquid-vapor transition.

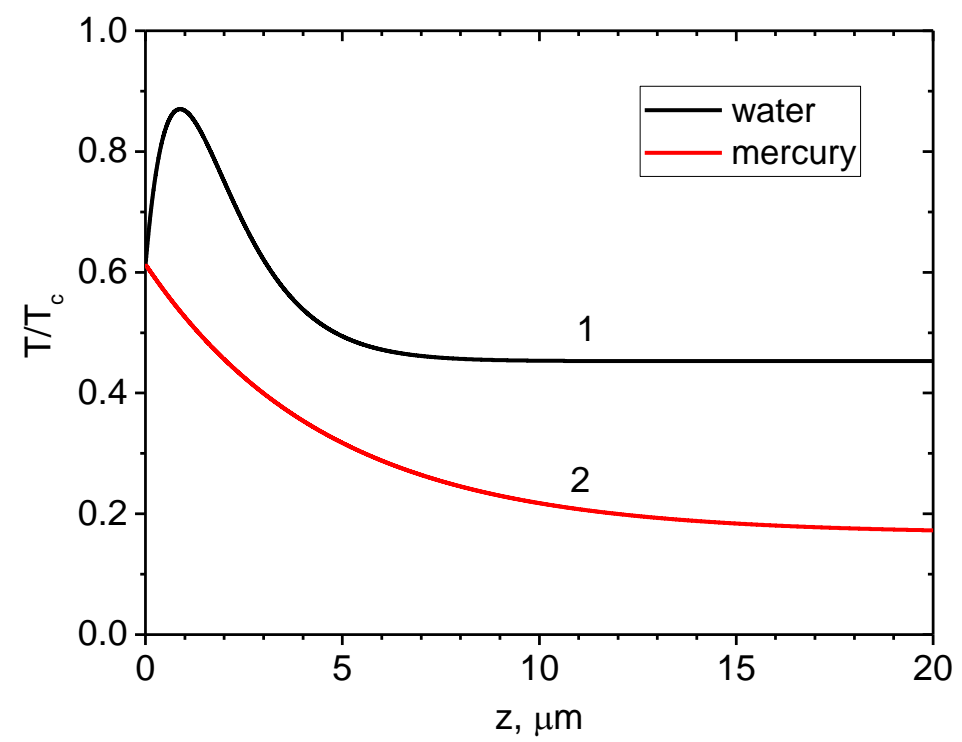

Figure 1: Steady-state temperature profiles normalized to critical temperature in water (curve 1) and in mercury (curve 2) at absorbed intensities of 40 and $660 \mathrm{~kW} / \mathrm{cm}^{2}$ respectively.

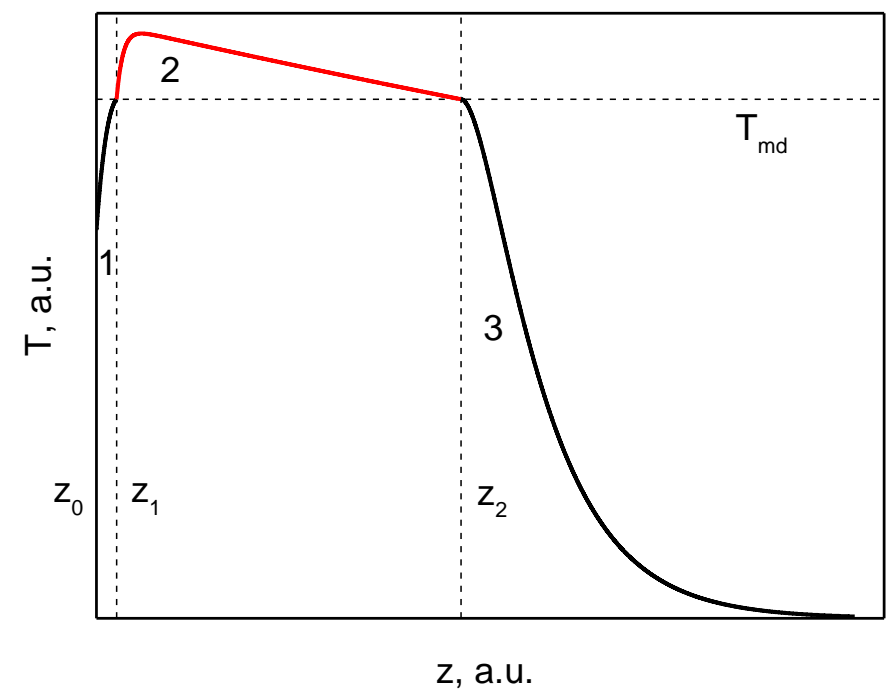

Figure 2: General form of steady-state temperature profile with a metal-dielectric transition in liquid metal: 1 - thin metal film on irradiated surface, 2 - dielectric layer, 3 - metal. 


\subsection{Steady-state regime of metal-dielectric transition in mercury}

In the case of metal-dielectric transition parameter $y$ is not constant in the steady-state regime and the temperature distribution has the form [7,8] which is shown schematically in Fig. 2. This not-in-scale representation of temperature distribution is necessary to mark explicitly boundaries between metal and dielectric layers which can have very different thickness in accordance with solution of equation (4). The solution has piecewise form including three different layers with thicknesses $H_{1}, H_{2}, H_{3} \gg H_{1}, H_{3} \gg H_{2}$.

In each layer $i$ with constant $\alpha_{i}$ and $\chi_{i}$ the temperature distribution $T_{i}(z)$ can be expressed as follows

$$
\begin{gathered}
T_{i}(z)=\mathrm{a}_{i} \exp \left[-\alpha_{i}\left(z-z_{i-1}\right)\right]+\mathrm{b}_{i} \exp \left[-\beta_{i}\left(z-z_{i-1}\right)\right]+\mathrm{c}_{i} \quad \text { for } \quad z_{i-1} \leq z \leq z_{i}, \quad i=1,2,3 \\
\mathrm{a}_{i}=I_{i-1} /\left[C V\left(1-y_{i}\right)\right], \quad \beta_{i}=V / \chi_{i}, \quad y_{i}=\alpha_{i} \chi_{i} / V, \quad z_{3} \rightarrow \infty
\end{gathered}
$$

The constants $\alpha_{i}$ and $\chi_{i}$ in metal layers are $\alpha_{1}=\alpha_{3}=\alpha_{m}, \chi_{1}=\chi_{3}=\chi_{m}$, and in dielectric layer are $\alpha_{2}=\alpha_{d}, \chi_{2}=\chi_{d}$. The absorbed intensity in each layer is determined by the recurrence relation $I_{i}$ $=I_{i-1} \exp \left(-\alpha_{i} H_{i}\right)$ where $I_{0}=I$ and layer thickness is $H_{i}=z_{i}-z_{i-1}$. At point $z_{0}=0$ boundary conditions (2) are used, and at the points $z_{1}$ and $z_{2}$ the following boundary conditions are used $[7,8]$.

$$
\begin{array}{rrrr}
T_{1}(z)=T_{m d}, & T_{2}(z)=T_{m d}, \quad \chi_{2} \partial T_{2} / \partial z=\chi_{1} \partial T_{1} / \partial z & \text { at } z=z_{1} \\
T_{2}(z)=T_{m d}, \quad T_{3}(z)=T_{m d}, \quad \chi_{3} \partial T_{3} / \partial z=\chi_{2} \partial T_{2} / \partial z & \text { at } z=z_{2} \\
T_{3}(z)=T_{\infty}, \quad \partial T_{3} / \partial z=0 \quad \text { at } z \rightarrow \infty &
\end{array}
$$

where $T_{m d}$ is a metal-dielectric transition temperature. Note that the last equality in (10) is valid due to the exponential form of (7).

The coefficients $\mathrm{b}_{i}, \mathrm{c}_{i}$ in (7) for the first (metal) layer is determined by the evaporative boundary conditions (2) and are given below

$$
\mathrm{b}_{1}=-L / C-I_{0} y_{1} /\left[C V\left(1-y_{1}\right)\right], \quad \mathrm{c}_{1}=T_{s}+L / C-I_{0} /(C V)
$$

The whole solution is obtained after the sequential determination of unknown layer thicknesses and coefficients $b_{i}, c_{i}$ in (7). The first parts of the conditions $(8,9)$ determine the thicknesses of a thin subsurface metal film $H_{1}$ and a dielectric layer $H_{2}$ through the transcendental equation

$$
\mathrm{a}_{j} \exp \left(-\alpha_{j} H_{j}\right)+\mathrm{b}_{j} \exp \left(-\beta_{j} H_{j}\right)+\mathrm{c}_{j}=T_{m d} \quad \text { for } j=1,2
$$

The equations for the temperature distribution coefficients in the second and third layers in (7) are obtained using the remaining conditions from $(8,9)$

$$
\begin{gathered}
\mathrm{a}_{j+1}+\mathrm{b}_{j+1}+\mathrm{c}_{j+1}=T_{m d}, \quad \text { for } j=1,2 \\
-\chi_{j+1}\left[\alpha_{j+1} \mathrm{a}_{j+1}+\beta_{j+1} \mathrm{~b}_{j+1}\right]=-\chi_{j}\left[\alpha_{j} \mathrm{a}_{j} \exp \left(-\alpha_{j} H_{j}\right)+\beta_{j} \mathrm{~b}_{j} \exp \left(-\beta_{j} H_{j}\right)\right] \equiv f_{j} \quad \text { for } j=1,2
\end{gathered}
$$

which give finally

$$
\mathrm{b}_{j+1}=-f_{j} / V-y_{j+1} \mathrm{a}_{j+1}, \quad \mathrm{c}_{j+1}=T_{m d}-\mathrm{a}_{j+1}-\mathrm{b}_{j+1} \quad \text { for } j=1,2
$$

It should be mentioned that $c_{3}=T_{\infty}$ is not independent parameter when values of $I, V$ and $T_{s}$ are given. This is due to energy conservation relation (5) or its special case which follows after integrating $T_{3}(z)$ from $z_{2}$ to $z \rightarrow \infty$ 


$$
I_{2}=V C\left(T_{m d}-T_{\infty}\right)+C f_{2}
$$

It is useful to note also that the distribution $T_{3}(z)$ in metal is monotonous in contrast to (6) because boundary condition at $z=z_{3}$ differs from the vaporization case (2).

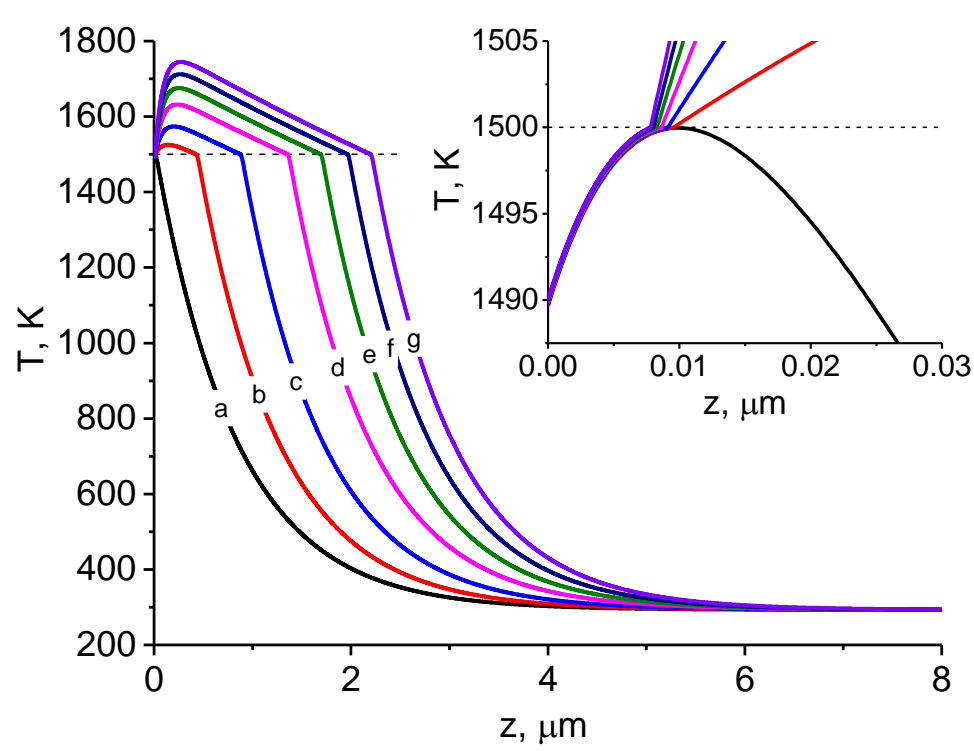

Figure 3: Steady-state temperature profiles in liquid mercury at various absorbed intensities (a-g) providing subcritical values of temperature maximum in dielectric layer for the case of $\alpha_{d}=10^{3} \mathrm{~cm}^{-1}$ and $I=4.221$ (a), 4.2215 (b), 4.222 (c), 4.223 (d), 4.224 (e), 4.225 (f), 4.226 (g) MW/cm².

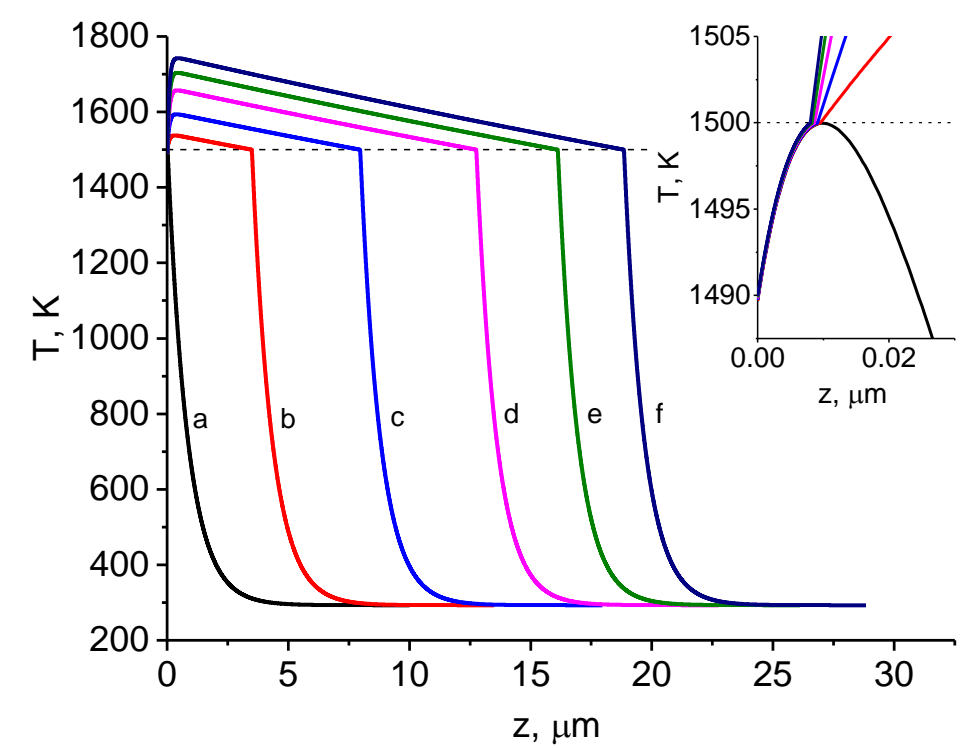

Figure 4: Steady-state temperature profiles in liquid mercury at various absorbed intensities (a-g) providing subcritical values of temperature maximum in dielectric layer for the case of $\alpha_{d}=10^{2} \mathrm{~cm}^{-1}$ and $I=4.221$ (a), 4.2215 (b), 4.222 (c), 4.223 (d), 4.224 (e), 4.225 (f) $\mathrm{MW} / \mathrm{cm}^{2}$. 
In metal case $y \gg 1$ and maximum value $T_{\max }$ in the temperature distribution (6) differs from $T_{s}$ but slightly. Temperature maximum $T_{\max }$ in dielectric layer can exceed $T_{m d}$ significantly as it is evident from curve 1 in Fig. 1 . From $T_{2}(z)$ distribution and the equation $\partial T_{2} / \partial z=0$ for $T_{\max }=T_{2}\left(z_{\max }\right)$ one obtains

$$
T_{\text {max }}=a_{2}\left(-\frac{b_{2} \alpha_{2}}{a_{2} \beta_{2}}\right)^{\frac{\beta_{2}}{\alpha_{2}-\beta_{2}}}+b_{2}\left(-\frac{b_{2} \alpha_{2}}{a_{2} \beta_{2}}\right)^{\frac{\alpha_{2}}{\alpha_{2}-\beta_{2}}}+c_{2}, \quad z_{\max }=z_{1}-\frac{\ln \left(-\frac{b_{2} \alpha_{2}}{a_{2} \beta_{2}}\right)}{\alpha_{2}-\beta_{2}}
$$

It is clear that maximum distribution temperature $T_{\max }$ should not exceed the thermodynamic stability limit (spinodal line) $T_{l}$ which is somewhat lower than the critical temperature $T_{c}$. One should keep in mind also that the difference $T_{c}-T_{m d}$ is small compared with $T_{c}$.

This condition results in rather strong limits on possible realization of such vaporization regime. Due to this condition the intensity interval $\Delta I_{m d}$ for realization of the steady-state vaporization regime with metal-dielectric transition is very narrow and its localization is close to the threshold intensity value $I_{t h}$.

\begin{tabular}{|c|c|}
\hline symbol & value \\
\hline$\alpha_{m}$ & $10^{6} \mathrm{~cm}^{-1}$ \\
\hline$\chi_{m}$ & $5.8 \times 10^{-2} \mathrm{~cm}^{2} / \mathrm{s}$ \\
\hline$\chi_{d}$ & $5.8 \times 10^{-3} \mathrm{~cm}^{2} / \mathrm{s}$ \\
\hline$\rho$ & $13.5 \mathrm{~g} / \mathrm{cm}^{3}$ \\
\hline$C$ & $1.9 \mathrm{~J} \mathrm{~cm}^{-3} \mathrm{~K}^{-1}$ \\
\hline$L$ & $3.8 \mathrm{~kJ} \mathrm{~cm}^{-3}$ \\
\hline$T_{m d}$ & $1500 \mathrm{~K}$ \\
\hline
\end{tabular}

Table 1 : The constant mercury parameters used in the calculation of the metal-dielectric transition.

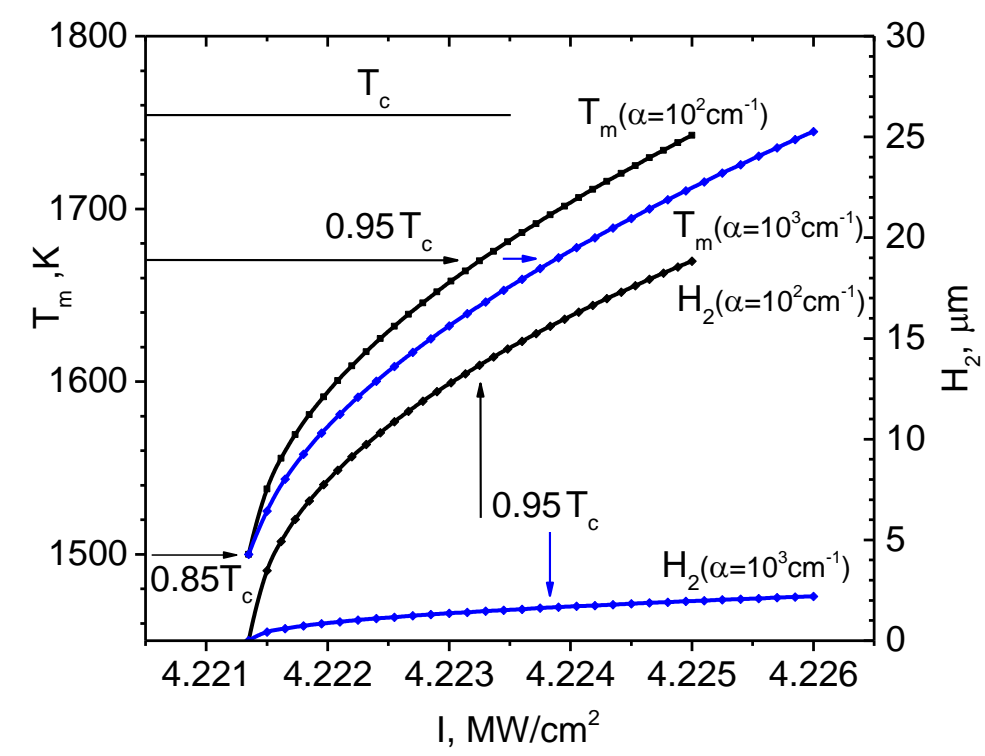

Figure 5: The dependences of steady-state dielectric layer thickness $\mathrm{H}_{2}$ and temperature maximum $T_{\mathrm{m}}$ on absorbed intensity $I$ in interval from dielectric layer threshold to critical temperature $T_{\mathrm{c}}$. 


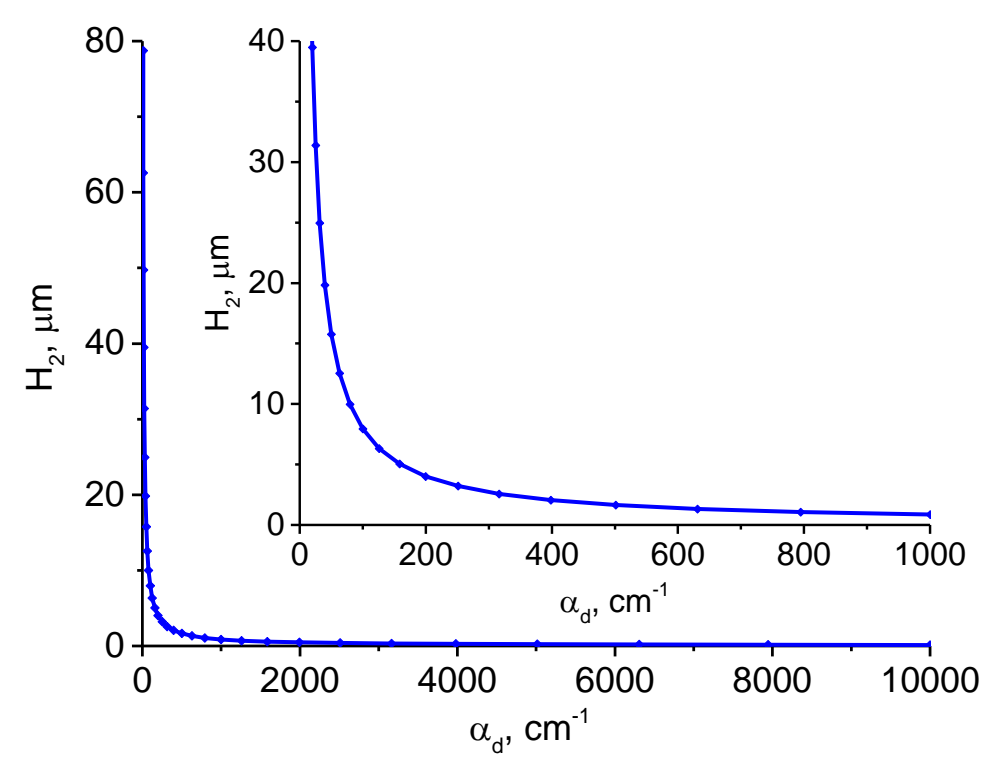

Figure 6: The dependence of steady-state dielectric layer thickness $H_{2}$ on absorption coefficient $\alpha_{\mathrm{d}}$ in this layer at constant absorbed intensity $I=4.222 \mathrm{MW} / \mathrm{cm}^{2}$.

The calculated temperature distributions in mercury are shown in Fig. 3, 4. The threshold intensity value is $I_{\text {th }}=4.2215 \mathrm{MW} / \mathrm{cm}^{2}$ while the intensity interval for realization of the steady-state vaporization regime with metal-dielectric transition $\Delta I_{m d}=4.5 \mathrm{~kW} / \mathrm{cm}^{2}$ (at $\alpha_{d}=$ $10^{3} \mathrm{~cm}^{-1}$ ) or $\Delta I_{m d}=3.5 \mathrm{~kW} / \mathrm{cm}^{2}$ (at $\alpha_{d}=10^{2} \mathrm{~cm}^{-1}$ ) is rather small $\Delta I_{m d}<<I_{t h}$.

Dependences of mercury dielectric layer thickness $H_{2}$ and temperature maximum $T_{m}$ on absorbed intensity $I$ in interval from dielectric layer threshold to critical temperature $T_{c}$ as well as dependence of dielectric layer thickness $H_{2}$ on absorption coefficient $\alpha_{d}$ at constant absorbed intensity $I=4.222 \mathrm{MW} / \mathrm{cm}^{2}$ are shown in Fig. 5 and 6.

In experiments the metal-dielectric transition in transient regime can be realized at higher intensities than $I_{t h} \sim 4.2 \mathrm{MW} / \mathrm{cm}^{2}$ for the steady-state case. In the transient regime the transition front velocity exceeds the vaporization front velocity and temperature maximum in the target attains the thermodynamic stability limit $T_{l}$. When this occurs the explosive boiling begins to develop in subnanosecond scale $[13,14]$.

\section{1-D ESTIMATIONS OF RECENT EXPERIMENTAL RESULTS}

In ref. [11] pressure behavior in liquid mercury is investigated during irradiation with different laser pulses: a train of several subnanosecond laser peaks divided with $8 \mathrm{~ns}$ intervals and a single $30 \mathrm{~ns}$ relatively smooth laser pulse. Using intensity modulated laser pulses permits to obtain information about irradiated surface displacement as it was first demonstrated in [15] for the case of dielectric liquids irradiated with harmonically modulated laser pulses. For metals laser intensity modulation due to mode-locking is more preferable than harmonic modulation due to two mode beating $[10,16]$.

It is shown in [11] that the pressure response detected with piezoelectric transducers as well as the surface displacement can not be explained in the framework of surface metal evaporation. In particular, significant diminishing $\delta$ of the acoustical arrival time is observed 
which means that the laser absorption zone deepens into the target for far more distance $h=$ $v_{s} \delta, v_{s}$ - sound velocity, than it is the case for metal ablation where $h_{m} \sim \alpha_{m}{ }^{-1}$.

Behavior of the kind may be related to the metal-dielectric transition [5-10] when its front moves deeply into the irradiated target. The transition modeling in [8] for supposed metaldielectric transition in $\mathrm{Al}$ shows additional bump in the pressure curve which is visible also in [11] at intensities $\geq 50 \mathrm{MW} / \mathrm{cm}^{2}$.

One can estimate the transition front velocity as $v_{f} \sim h / \tau=v_{s} \delta / \tau$ which exceeds sound velocity $v_{s}$ if $\delta>\tau$. In experimental results [11] $\delta / \tau \sim 2$ which means supersonic movement of the transition front.

From energy balance equation it follows that to realize such movement it is necessary to have the absorbed laser intensity at the transition front determined approximately by the expression $I=v_{f} C\left(T_{m d}-T_{\infty}\right)$. For $v_{f} \sim 2 v_{s}=2.8 \mathrm{~km} / \mathrm{s}, C=1.9 \mathrm{~J} / \mathrm{cm}^{3}, T_{m d}-T_{\infty}=1200 \mathrm{~K}$ this gives $I=0.6 \mathrm{GW} / \mathrm{cm}^{2}$. This intensity value exceeds the value in experiment [10] which is lower than $0.1 \mathrm{GW} / \mathrm{cm}^{2}$. The estimated pressure generated during such front movement also far exceeds the maximum pressure value observed in [11] which is about one kbar while the 1-D estimation gives about a hundred kbar.

A possible reason of such discrepancies is probably due to violation of 1-D approach in the considered case [11] where intensity distribution is not constant over the irradiation spot. The initial Gaussian distribution over the irradiation spot can transform itself to more sharp distribution during its propagation in the target where the metal-dielectric transition occurs.

\section{CONCLUSIONS}

In the framework of simplified 1-D heat conduction approach it is shown that the steadystate vaporization regime of mercury with supposed metal-dielectric transition can occur only in very narrow irradiation intensity interval. Analysis of the recent experimental results [11] of mercury laser ablation shows remarkable discrepancy between the 1-D theoretical estimation and experiment. The discrepancy can be probably due to violation of 1-D approach applicability in the considered case because possibility of significant transformation of initial Gaussian intensity distribution over the irradiation spot. For this reason, it is interesting to investigate laser ablation of mercury with constant laser intensity distribution over the irradiated spot as well as to study stability of this regime and to model the ablation process with metal-dielectric transition in the framework of continual 2-D approach or molecular dynamic calculations.

\section{REFERENCES}

[1] A.L. Khomkin and A.S. Shumikhin, "Critical Points of Metal Vapors", JETP, 121, 521-528 (2015).

[2] V.S. Vorob'ev and E.M. Apfelbaum, "The Generalized Scaling Laws Based on Some Deductions from the van der Waals Equation", High Temperature, 54, 175-185 (2016).

[3] Ch. Wu and L.V. Zhigilei, "Microscopic Mechanisms of Laser Spallation and Ablation of Metal Targets from Large-Scale Molecular Dynamics Simulations", Appl. Phys. A., 114, 11-32 (2014).

[4] I. Iosilevskiy, V. Gryaznov, "Uranium critical point location problem", Zababakhin Scientific Talks, International Conference March 18-22, 95 (2019).

[5] L.D. Landau, Ya.B. Zeldovich, "On the Relation between the Liquid and the Gaseous States of Metals", Acta Physicochim. USSR, 18, 194-197 (1943).

[6] V.A. Batanov, F.V. Bunkin, A.M. Prokhorov, V.B. Fedorov, "Evaporation of Metallic Targets 
Caused by Intense Optical Radiation", JETP, 36(2), 311-322 (1973).

[7] R.V. Karapetyan and A.A. Samokhin, "Influence of an increase in the transparency on the intense evaporation of metals by optical radiation", Sov. J. Quantum Electron., 4, 1141-1142 (1975).

[8] S.N. Andreev, V.I. Mazhukin, N.M. Nikiforova, A.A. Samokhin, "On possible manifestations of the induced transparency during laser evaporation of metals", Quantum Electronics, 33, 771-776 (2003).

[9] N.E. Bykovsky, S.M. Pershin, A.A. Samokhin and Yu.V. Senatsky, "Transmittance jump in a thin aluminium layer during laser ablation", Quantum Electron., 46(2), 128-132 (2016).

[10] A.A. Samokhin, S.I. Kudryashov, A.E. Zubko, and A.V. Sidorin, "Modelling of nanosecond laser ablation. Continual approach", Math. Montis., 37, 76-90 (2016).

[11] A.A. Samokhin, E.V. Shashkov, N.S. Vorobiev, A.E. Zubko, "On acoustical registration of irradiated surface displacement during nanosecond laser-metal interaction and metal-nonmetal transition effect", Applied Surface Science, (2020, in press), DOI: 10.1016/j.apsusc.2019.144261

[12] A.A. Samokhin, "First-order phase transitions induced by laser radiation in absorbing condensed matter", in Effect of laser radiation on absorbing condensed matter, V.B. Fedorov (Ed.), Nova Science Publishers, (1990).

[13] V.I. Mazhukin, A.A. Samokhin, A.V. Shapranov and M.M. Demin, "Modeling of thin film explosive boiling-surface evaporation and electron thermal conductivity effect", Mater. Res. Express, 2, 016402 (2015), DOI: 10.1088/2053-1591/2/1/016402

[14] A.A. Samokhin, V.I. Mazhukin, M.M. Demin, A.V. Shapranov, A.E. Zubko, "Molecular dynamics simulation of al explosive boiling and transcritical regimes in nanosecond laser ablation", Math. Montis., 41, 55-72 (2016).

[15] A.A. Samokhin and N.N. Il'ichev, "On photoacoustic monitoring of laser evaporation front movement", Quantum Electronics, 40(8), 659-660 (2010).

[16] A.E. Zubko and A.A. Samokhin, "Pressure pulses generated in metals under the action of nanosecond laser pulses with periodically modulated intensity", Engineering Physics, 3, 47-52 (2017); (the main text is in Russian).

Received November 20, 2019 